\author{
Johannes Geyer, Peter Haan, C. Katharina Spieß \& \\ Katharina Wrohlich
}

\title{
Das Elterngeld und seine Wirkungen auf das Haushaltseinkommen junger Familien und die Erwerbstätigkeit von Müttern
}

\section{The new German parental leave benefit scheme and its effects on young families' household income and on mothers' paid employment}

\begin{abstract}
Zusammenfassung:
Mit der Einführung des Elterngeldes im Jahr 2007 beabsichtigte die Bundesregierung die Bedingungen für Familien mit jungen Kindern zu verbessern. Die neue familienpolitische Leistung hatte mehrere Ziele, von denen in diesem Beitrag drei zentrale untersucht werden: Zum Ersten sollte für Eltern in der Frühphase der Elternschaft ein Schonraum geschaffen werden. Zum Zweiten ist es ein erklärtes Ziel des Elterngeldes, es beiden Elternteilen zu ermöglichen, ihre wirtschaftliche Existenz eigenständig zu sichern, und drittens soll die Erwerbstätigkeit von Müttern gefördert werden. In diesem Beitrag wird anhand einer empirischen Wirkungsstudie überprüft, ob diese Ziele erreicht wurden. Die Ergebnisse zeigen, dass Familien im ersten Jahr nach der Geburt durch das Elterngeld durchschnittlich etwa 480 Euro im Monat mehr haben als vor seiner Einführung. Außerdem zeigt sich, dass die Erwerbstätigkeit von Müttern in diesem Zeitraum zurückgegangen ist. Mütter mit Kindern im zweiten Lebensjahr haben aufgrund der Einführung des Elterngeldes eine höhere Wahrscheinlichkeit, in den Beruf zurückzukehren.
\end{abstract}

Schlagwörter: Einkommenseffekte, Arbeitsangebot von Müttern, Elternzeit, Elterngeld, Erziehungsgeld, Mikrosimulation

\begin{abstract}
:
In 2007, Germany introduced a new parental leave benefit scheme, the so-called "Elterngeld". The new benefit is an income-related transfer and is granted for a maximum period of 14 months. The more generous, but shorter Elterngeld replaced the former means-tested flat rate benefit that could be drawn for up to 24 months. One of the aims of the reform was to smooth household income in the year after childbirth and another to increase the incentives to return quickly to the labour market. In this study, we analyse empirically the change in income in the first year after birth and the effect of increasing incentives for mothers to return to the labour market in the second year after childbirth. We find that the average increase of net household income of families with a child in the first year after birth amounts to 480 euro per month. With respect to labour supply, we show that in the first year after childbirh, mothers' labour supply decreases. In the second year labour supply of mothers in East Germany and low-income mothers in both parts of Germany increases.
\end{abstract}

Key words: income effects, maternal labour supply, parental leave, micro simulation study 


\section{Einleitung}

Schwierigkeiten bei der Vereinbarkeit von Familie und Beruf sind in Deutschland nach wie vor entscheidende Faktoren für niedrige Erwerbsquoten von Müttern mit sehr jungen Kindern - dies wird besonders deutlich, wenn die Erwerbsquoten mit anderen europäischen Ländern verglichen werden. Die Reformen der deutschen Familienpolitik der vergangenen Jahre zielten deshalb unter anderem darauf, diese Vereinbarkeit zu verbessern (vgl. z.B. Spieß 2011). Eine dieser Reformen ist das einkommensabhängige Elterngeld, dessen bundesweite Einführung für Geburten ab dem 1.1.2007 das bedürftigkeitsgeprüfte Erziehungsgeld abgelöst hat. Das Elterngeld ist eine zentrale Leistung der ,nachhaltigen Familienpolitik“, wie sie von der damaligen schwarz-roten Bundesregierung verstanden wurde (vgl. z.B. Ristau 2005). Mit dem Elterngeld wurden mehrere Ziele verfolgt. Bujard (in diesem Heft) identifiziert die folgenden fünf Ziele: (1) Einkommenssicherung in der Familiengründungsphase, (2) einen Schonraum im ersten Jahr ermöglichen, (3) Erwerbsbeteiligung von Müttern fördern, (4) Fürsorgebeteiligung von Vätern fördern und (5) ein valentes Ziel, die Familiengründung erleichtern. Gegenstand dieses Beitrags ist es, das Elterngeld in Hinblick auf ausgewählte Ziele, nämlich seine Einkommenswirkung im ersten Jahr nach der Geburt eines Kindes und die Wirkung auf das Arbeitsangebotsverhalten von Müttern im ersten und zweite Jahr nach der Geburt zu untersuchen.

Ohne hier auf alle Neuerungen des Elterngeldes im Einzelnen einzugehen (vgl. dazu ausführlicher z.B. Wrohlich et al. 2012), sollen vorab einige wichtige Elemente des Elterngeldes hervorgehoben werden, die für die folgende Analyse von Bedeutung sind. Bei seiner Einführung lag die Höhe des Elterngelds bei $67 \%$ des letzten Jahresnettoentgelts mit einem Maximalbetrag von 1.800 Euro und einer Mindestleistung von 300 Euro (,Sockelbetrag“) im ersten Lebensjahr des Kindes. Dadurch verbessert sich im Vergleich zum Erziehungsgeld die Einkommenssituation von Familien in diesem Zeitraum, wenn die Mutter vorher erwerbstätig war. Aufgrund der Bedürftigkeitsprüfung waren viele dieser Haushalte, die nun einen Elterngeldanspruch haben, vorher nicht leistungsberechtigt. Gleichzeitig stellt der Sockelbetrag weitgehend sicher, dass sich Familien im Vergleich zum früheren Erziehungsgeld in dieser Zeit nicht schlechterstellen. ${ }^{1}$ Die maximale Bezugsdauer wurde von 2 Jahren auf 12 bzw. 14 Monate (wenn beide Elternteile Elterngeld beziehen) verkürzt. Dadurch stellen sich im zweiten Lebensjahr eines Kindes Familien schlechter, die einen Anspruch auf Erziehungsgeld gehabt hätten. In erster Linie sind dies einkommensschwache Haushalte. Während sich im ersten Lebensjahr die Erwerbsanreize tendenziell verringern, erhöhen sie sich im zweiten Jahr für Mütter, die nun keinen Anspruch auf entsprechende staatliche Leistungen haben. Im Gegensatz zu früheren Reformen des Erziehungsgeldes, beinhaltete die Einführung des Elterngeldes erstmalig eine Verkürzung der Transferperiode und damit eine Reduzierung der Anreize für Mütter länger aus dem Arbeitsmarkt auszuscheiden. Gleichzeitig wurden die Anreize für mindestens ein Jahr nicht bzw. reduziert erwerbstätig zu sein, deutlich gesteigert. Diese Veränderung der Anreize und der damit verbundenen Wirkungen des Elterngelds auf das Haushaltseinkommen und die Erwerbsbeteiligung von Müttern werden wir im Folgenden untersuchen.

1 Allerdings unterliegt das Elterngeld im Gegensatz zum Erziehungsgeld dem Progressionsvorbehalt, was dazu führen kann, dass sich einige wenige Familien schlechter stellen. 
Die Einkommenswirkungen ergeben sich aus der neuen Berechnungsweise des Elterngeldes und können unter Absehung von Verhaltensanpassungen unmittelbar unter Verwendung repräsentativer Haushaltsdaten berechnet bzw. simuliert werden. Bei der Reaktion des Arbeitsangebots stützen wir unsere empirischen Untersuchungen auf ein Standard-Arbeitsangebotsmodell, welches die Änderungen in den finanziellen Anreizen als zentralen Verhaltensparameter modelliert. Dieses ökonomische Modell reduziert das Arbeitsangebot der Mutter auf ein Kosten-Nutzen-Kalkül bezüglich des Trade-offs von Freizeit und Erwerbseinkommen. Damit soll ein wichtiger Aspekt der Erwerbsentscheidung herausgearbeitet werden, ohne dass wir den Anspruch erheben, dass das Modell alle Facetten der Erwerbsentscheidung berücksichtigt. Einschränkend ist darüber hinaus festzuhalten, dass wir in diesem Beitrag nur die kurzfristigen Wirkungen der Reform auf die Erwerbsbeteiligung von Müttern und das Haushaltseinkommen analysieren können. Es ist zu vermuten, dass es im Zuge der Reform zum Beispiel zu Veränderungen der sozialen Normen bezüglich der Erwerbsbeteiligung von Müttern mit jungen Kindern kommt. Diesen langfristigen Einfluss können wir mit den von uns gewählten Methoden nicht untersuchen. Zudem wurden mit den zwei „Partnermonaten“ zusätzlich Anreize geschaffen, die Elternzeit unter den Eltern aufzuteilen (vgl. dazu z.B. Schulz/Rost 2012). Auch dies kann Müttern den Wiedereinstieg in den Arbeitsmarkt erleichtern und langfristig zu einer Änderung der Väterbeteiligung an der Familienarbeit beitragen. Ein weiterer Aspekt der Reform ist, dass das Elterngeld Anreize setzt, vor der Geburt eines Kindes erwerbstätig zu werden, um ein höheres Elterngeld zu erzielen. Diese Veränderung der Erwerbsanreize ist ebenfalls nicht Gegenstand dieses Beitrags.

Unser Beitrag ist wie folgt aufgebaut: Nach einem Überblick über bisherige empirische Studien zu den hier fokussierten Zielen von Elterngeld- und Elternzeitregelungen, diskutieren wir die Daten und methodischen Vorgehensweisen unserer eigenen empirischen Untersuchung. ${ }^{2}$ Daran schließt sich die Darstellung und Diskussion der Ergebnisse an. Der Beitrag schließt mit familien-, bildungs- und arbeitsmarktpolitischen Schlussfolgerungen.

\section{Bisherige Forschungsarbeiten}

Die Wirkungen von Elterngeld- und Elternzeitregelungen bzw. der damit verbundenen Transfer- und Lohnersatzleistungen auf die Erwerbstätigkeit von Müttern mit jungen Kindern wurden in einer Vielzahl von empirischen Arbeiten aus den USA und Europa untersucht. ${ }^{3}$ Dabei wurde in der Regel auf die Erwerbstätigkeit und die langfristigen Folgen ei-

2 Die hier dargestellten Ergebnisse der eigenen Analysen beruhen auf Wrohlich et al. (2012). Die Ergebnisse hinsichtlich der Erwerbseffekte sind auch in Geyer et al. (2012) zusammengefasst. Den Analysen liegt der so genannte „Elterngeldmonitor“ zugrunde. Das Bundesministerium für Familie, Senioren, Frauen und Jugend, welche den Elterngeldmonitor in Auftrag gegeben hat, hat die Ergebnisse für eine breite Fachöffentlichkeit zusammengefasst (BMFSFJ 2012).

3 Für eine zusammenfassende Darstellung vgl. auch Spieß (2011). Dabei unterscheiden diese Studien nicht immer zwischen den Effekten die auf der einen Seite auf Elterngeldregelungen im engeren Sinne und auf der anderen Seite auf Elternzeitregelungen zurückzuführen sind. 
ner Erwerbsunterbrechung für spätere Verdienstmöglichkeiten fokussiert. Sehr viel weniger wurde die Wirkung dieser familienpolitischen Maßnahmen auf die wirtschaftliche Stabilität von Familien analysiert - dieser Effekt wird in der Regel nur über Veränderungen in der Lohnentwicklung von Müttern abgebildet. Einige wenige Arbeiten, insbesondere skandinavische, adressieren die Frage, inwiefern entsprechende Regelungen zu einer größeren Geschlechtergerechtigkeit führen, indem sie z.B. dazu beitragen, dass mehr Väter Elternzeit in Anspruch nehmen (vgl. dazu z.B. Ekberg et al. 2013, Duvander/Andersson 2006 und Oláh 2003 für Arbeiten aus dem skandinavischen Raum sowie Geissler/Kreyenfeld 2011, Schulz/ Rost 2012 und Schober 2011 für deutsche Studien bzw. einen deutsch-britischen Vergleich). Hier handelt es sich vielfach um Arbeiten, die sich auf soziologische Erklärungsansätze stützen. Ein weiterer Strang der Literatur untersucht explizit, inwiefern eine Ausdehnung der Elternzeit und/oder des Elterngeldbezugs dem Ziel einer frühen Förderung entgegenkommt, da Eltern dann mehr Zeit mit ihren Kindern verbringen können - hier sind primär Arbeiten aus dem bildungs- und familienökonomischen Bereich zu finden. Auf der Basis makroökonometrischer Ansätze analysieren dies Ruhm (2000) und Tanaka (2005), während zu den mikroökonometrischen Arbeiten die Studien von Baker und Milligan (2008), Würtz-Rasmussen (2010), Liu und Nordström Skans (2010) oder die deutsche Studie von Dustmann und Schönberg (2012) zählen. Dustmann und Schönberg (2012) können in Analogie zu den anderen europäischen Arbeiten keinen statistisch messbaren Effekt für Kinder, deren Mütter länger in Elternzeit gehen, feststellen. Einige wenige Studien untersuchen die Wirkung von entsprechenden Regelungen auf das Geburtsverhalten (vgl. dazu z.B. Lalive/Zweimüller 2009). Diesen mikrodatenbasierten Arbeiten gemeinsam ist, dass sie vorrangig auf die Erfassung sozioökonomischer Faktoren abstellen und mehrheitlich Einstellungsfragen ${ }^{4}$ bzw. Fragen kultureller Muster auBen vor bleiben.

Die Wirkungsforschung zu den Erwerbs- und Einkommenseffekten von Elterngeldund Elternzeitregelungen basiert zum einen auf makroökonometrischen Arbeiten, wie z.B. von Ruhm und Teague (1997) sowie Ruhm (1998). Die Arbeit von Ruhm (1998), die neun europäische Länder für die Jahre 1969 bis 1993 untersucht, legt u.a. nahe, dass sich eine längere und bezahlte Elternzeit positiv auf die Erwerbsquote, aber negativ auf die relativen durchschnittlichen Löhne von Frauen im gebärfähigen Alter auswirkt. Dieser Effekt lässt sich so erklären, dass die betrachteten Transfers i.d.R. abhängig von einer vorhergehenden Erwerbstätigkeit sind. Zum anderen wird in mikroökonometrischen Studien untersucht, inwiefern Veränderungen oder regionale Unterschiede in Elternzeitregelungen das Arbeitsangebot von Müttern mit jungen Kindern beeinflussen. Für US-amerikanische Arbeiten, siehe z.B. Berger und Waldvogel (2004), Hashimoto et al. (2004) und Han et al. (2007). Diese Studien weisen allerdings keine eindeutigen Ergebnisse in Hinblick auf die Wirkung von entsprechenden Regelungen auf. Europäische Arbeiten, wie z.B. Pylkkänen und Smith (2004) oder Würtz-Rasmussen (2010) untersuchen dänische und schwedische Regelungen. Pylkkänen und Smith (2003) zeigen, dass die bezahlte Erziehungszeit eine wichtige Determinante des Wiedereintritts in Beschäftigung ist. Es zeigt sich aber zudem, dass auch die Infrastruktur von Betreuungseinrichtungen dann eine wichtige Rolle spielt,

$4 \mathrm{Zu}$ diesen Einstellungen gehört beispielsweise auch die Akzeptanz einer außerfamilialen Kinderbetreuung, welche in Deutschland sehr unterschiedlich ausfällt (vgl. dazu Bujard et al. 2012). 
wenn es nicht genügend Betreuungsplätze gibt. Ein EU-Vergleich zwischen 5 Ländern wird von Gutierrez-Domenech (2005) durchgeführt. Hier zeigt sich ebenfalls die Bedeutung von unterschiedlichen institutionellen Rahmenbedingungen und der Arbeitsmarktsituation von Müttern. Hervorzuheben ist auch die Arbeit von Lalive und Zweimüller (2009), die auf der Basis österreichischer Sozialversicherungsdaten eine in Österreich 1990 einsetzende Verlängerung der Elternzeit und damit verbundener Transferzahlungen von einem auf zwei Jahre und eine Verkürzung derselben im Jahr 1996 auf 18 Monate untersucht. $^{5}$ Die Einführung der Elterngeld- und -zeitreformen erlaubt es, die Wirkung der Reform im Rahmen eines natürlichen Experiments zu untersuchen. Kurzfristig zeigt sich, dass eine Ausdehnung der Elternzeit zu einer geringeren Rückkehrwahrscheinlichkeit und zu Lohnabschlägen führt - langfristig sind die Effekte allerdings gering. Es sind keine bedeutenden Unterschiede für einzelne Lohngruppen festzumachen. Auch in einer neuen Analyse dieser Reformen von Lalive et al. (2011) zeigt sich, dass keine bedeutsamen mittel- bis langfristigen Lohneffekte zu finden sind.

Deutsche Wirkungsstudien fokussieren auf die vielfältigen Änderungen beim deutschen Erziehungsgeld seit den 1980er Jahren. Es werden Änderungen der maximalen Dauer des Erziehungsurlaubs bzw. der Elternzeit und der Teilzeitmöglichkeiten untersucht. Änderungen im Erziehungsgeld bzw. Elterngeld beziehen sich in der Regel auf dessen maximale Bezugsdauer, Einkommensgrenzen und die maximale Höhe. Die makroökonometrische Arbeit von Merz (2005) basiert auf Zeitreihen des Mikrozensus. Sie belegt, dass über die Jahre der Anteil von Frauen, die während der Elternzeit einer Teilzeiterwerbstätigkeit nachgehen, signifikant zugenommen hat. $\mathrm{Zu}$ den mikroökonometrischen Studien zählen die Arbeiten von Ondrich und Spiess (Ondrich et al. 1996, 1999, 2003a, 2003b). Sie zeigen eine Verringerung der Rückkehrwahrscheinlichkeiten mit zunehmender Ausdehnung des Erziehungsurlaubs. Dies hat auch Auswirkungen auf die Lohnentwicklung von Müttern: Beispielsweise verringert jeder zusätzliche Monat Erziehungsurlaub über 5 Jahre hinweg das Lohnwachstum um 1,5 Prozent (Ondrich et al. 2003b). Schönberg und Ludsteck (2007) bestätigen den negativen Zusammenhang zwischen der Dauer des Erziehungsurlaubs und dem Anteil der Mütter, die wieder erwerbstätig werden. Darüber hinaus kehrten viele Mütter mit dem Ende der Bezugsdauer des Erziehungsgeldes auf den Arbeitsmarkt zurück. Allerdings sind längerfristig geringere Effekte zu beobachten.

Auch die Einführung des deutschen Elterngelds im Jahr 2007 wurde bereits von anderen Autorenteams evaluiert. Im Rahmen einer Ex-ante-Evaluationsstudie haben Spiess und Wrohlich (2008) die Reform auf der Basis der Daten des Sozio-oekonomischen Panels (SOEP) simuliert. Ihre Simulation kommt zu dem Ergebnis, dass die Partizipationsquote von Müttern mit Kindern im zweiten Lebensjahr um 3 Prozentpunkte auf knapp 40 Prozent ansteigen wird. Kluve und Tamm (2013) haben in einer Ex-post Analyse die Reform auf der Basis einer eigenen Erhebung bei AOK-Versicherten der AOK Rheinland und Sachsen-Anhalt evaluiert. Sie schätzen den Rückgang in der durchschnittlichen Erwerbsquote von Müttern im ersten Jahr auf 6,5 Prozentpunkte. Für das zweite Jahr nach der Geburt schätzen sie keinen Anstieg der durchschnittlichen Erwerbswünsche, aller-

5 Diese Arbeit auf der Basis einer österreichischen Reform ist für diesen Beitrag deshalb von besonderem Interesse, da das österreichische System dem deutschen familienpolitischen System relativ ähnlich ist. 
dings zeigt sich ein positiver Effekt für Frauen aus Ostdeutschland, Frauen, die vor der Geburt nicht erwerbstätig waren, und für Mütter, die bereits Kinder hatten. Riphahn und Bergemann (2011) untersuchen ebenfalls die Wirkungen des Elterngeldes auf die Erwerbswünsche von Müttern. Der allgemeine Erwerbswunsch wird durch die Reform nicht verändert, allerdings steigt der Anteil von Müttern, die aufgrund der Reform schneller in den Arbeitsmarkt zurückkehren wollen, signifikant an.

\section{Eigene empirische Analysen}

In den eigenen Analysen fokussieren wir auf die Wirkung des Elterngeldes in Hinblick auf das Haushaltseinkommen und die Erwerbstätigkeit der Mütter. Die empirische Identifikation dieser Effekte beinhaltet verschiedene Probleme. So sind z.B. die Verteilungswirkungen des Elterngeldes auch davon abhängig, wie Eltern auf die Reform reagieren und ihr Verhalten anpassen. Dasselbe gilt für die Schätzung der Reformeffekte auf die Erwerbstätigkeit der Mutter, denn hier könnten Selektionseffekte den tatsächlichen Reformeffekt überzeichnen. Es könnte z.B. sein, dass sich aufgrund des Elterngeldes mehr Frauen mit höherer Erwerbsneigung entscheiden, Kinder zu bekommen. Bei der Abschätzung der Einkommensund Beschäftigungseffekte ist es deswegen notwendig ein Verfahren zu wählen, das die Effekte der Reform tatsächlich isolieren kann. Unsere empirischen Schätzungen basieren deswegen auf Müttern, die noch nicht vom Elterngeld profitieren konnten und demnach keine Anreize hatten ihr Verhalten aufgrund der Reform zu verändern.

Zur Analyse der Einkommens- und Beschäftigungseffekte der Einführung des Elterngeldes, gehen wir in zwei Schritten vor. Zunächst simulieren wir mit einem Mikrosimulationsmodell das kontrafaktische Einkommen von Müttern, die vor dem Jahr 2007 ein Kind unter zwei Jahren hatten. Damit können wir die Einkommenseffekte der Reform auf Basis eines ökonometrisch geschätzten Arbeitsangebotsmodells die Auswirkungen auf die Erwerbsbeteiligung von Müttern untersuchen.

\subsection{Datenbasis und methodisches Vorgehen}

Um die Auswirkungen der Einführung des Elterngeldes auf das Einkommen von jungen Familien zu untersuchen, verwenden wir ein Mikrosimulationsmodell. ${ }^{6}$ Dieses Modell ermöglicht die Berechnung von Nettoeinkommen für sämtliche Haushalte eines Mikrodatensatzes. Die Datengrundlage unserer Analysen ist das SOEP (Wagner et al. 2007, www. diw/soep), eine für Deutschland repräsentative Haushalts- und Personenbefragung. Für die Berechnung der Nettoeinkommen im Mikrosimulationsmodell benötigen wir die Informationen zu den Bruttoeinkünften aller Haushaltsmitglieder und die steuer- bzw. trans-

6 Für diese Analyse verwenden wir das Mikrosimulationsmodell STSM des DIW Berlin (vgl. Steiner et al. 2012). Das STSM beinhaltet die wichtigsten Elemente des deutschen Steuersystems (Ehegattensplitting, Kindergeld bzw. Kinderfreibetrag etc.), Sozialversicherungsbeiträge und alle wesentlichen Transfers wie Arbeitslosengeld, Arbeitslosenhilfe bzw. Arbeitslosengeld II, Wohngeld, Erziehungsgeld bzw. Elterngeld und Kinderzuschlag. 
ferrelevanten Charakteristika der Haushalte. Auf dieser Grundlage können die Nettoeinkommen sowohl für das geltende Recht als auch für andere, hypothetische Szenarien berechnet werden. Wir berechnen für alle Haushalte der Jahre 2004 bis 2006 die Nettoeinkommen für das geltende Recht des Jahres 2006 (Erziehungsgeld) und die hypothetischen Nettoeinkommen für das Recht des Jahres 2007 (Elterngeld). ${ }^{\top}$

Im ersten Schritt führen wir eine statische Analyse durch, das bedeutet, wir abstrahieren zunächst von möglichen Verhaltensänderungen der Haushalte in Bezug auf ihr Erwerbsverhalten. Diese Methode erlaubt es, den Einkommenseffekt des Elterngeldes zu ,isolieren“, d.h. die Veränderung der Einkommen von Familien aufgrund des Elterngeldes vor Verhaltensanpassung darzustellen. Damit können wir die hypothetische Frage, ,über welches Einkommen hätten diese Haushalte verfügt, hätte es das Elterngeld bereits 2006 gegeben?", unter der Annahme, dass die Haushalte ihr Verhalten nicht ändern, beantworten.

Um den kausalen Effekt des Elterngeldes auf die Erwerbsentscheidung von Müttern zu untersuchen, schätzen wir im nächsten Schritt ein strukturelles Arbeitsangebotsmodell. Dieses Modell basiert auf der mikroökonomischen Theorie, nach der Individuen jene Kombination aus Arbeitszeit, Haushaltseinkommen und Freizeit wählen, die ihnen den höchsten Nutzen stiftet. Entscheidend ist dabei die Abwägung zwischen zusätzlichem Nettoeinkommen und der Aufgabe von Freizeit. Natürlich stellt dieses Modell eine extreme Vereinfachung der Realität dar, letztlich wird die Entscheidung auf ein einfaches Kosten-Nutzen Kalkül der Mutter reduziert. Es hat sich gezeigt, dass diese einfache Approximation das Arbeitsangebotsverhalten relativ gut beschreiben kann und viele extrem aufwendige Erweiterungen des Modells (beispielsweise die Integration von Kinderbetreuungskosten, unfreiwillige Arbeitslosigkeit oder auch dynamische Effekte und Pfadabhängigkeit) die Ergebnisse qualitativ gar nicht und quantitativ nur in geringem Maße ändern (vgl. die Diskussion in Bach et al. 2012). Unabhängig davon vernachlässigt dieses Modell notwendigerweise andere Faktoren wie gesellschaftliche Erwartungen oder kulturelle Einstellungen der Personen. Dementsprechend ist es nicht Anspruch des Modells alle Aspekte zu beinhalten, die bei der Entscheidung über die Erwerbstätigkeit nach der Geburt relevant sind. Umgekehrt legen wir den Fokus auf einen aus unserer Sicht zentralen Verhaltensparameter. Darüber hinaus darf auch nicht vergessen werden, dass die Arbeitsangebotsentscheidung in einem institutionellen Rahmen stattfindet, den wir im Modell als gegeben unterstellen. Letztlich ist aber anzunehmen, dass die Größe der geschätzten Effekte auch von diesen Rahmenbedingungen abhängt. So kann die Entscheidung über die Erwerbstätigkeit beispielsweise vom Vorhandensein von Kinderbetreuungseinrichtungen abhängig sein.

Die Arbeitsangebotsentscheidung wird ökonometrisch als diskretes Auswahlmodell abgebildet, d.h. jede Mutter kann aus einer diskreten Anzahl von Arbeitszeitkategorien (Nicht-Erwerbstätigkeit, Teilzeit-Erwerbstätigkeit und Vollzeit-Erwerbstätigkeit) wählen. Konkret wird ein konditionales Logit-Modell geschätzt, mit dem die Wahrscheinlichkeit für jede Arbeitszeitkategorie bestimmt werden kann (Greene 2003: 719ff.). Um das Arbeitsangebotsmodell zu schätzen, muss deswegen zunächst das Netto-Haushaltseinkommen für jeden Haushalt in jeder Arbeitszeitkategorie auf Basis des Mikrosimulationsmo-

7 Die Datenbasis für unsere empirische Analyse sind alle SOEP-Haushalte aus den Wellen 20022009, in denen Mütter leben, die in diesem Beobachtungszeitraum ein Kind bekommen haben. Der Datensatz enthält sowohl alleinstehende, verheiratete und unverheiratete aber mit ihrem Partner zusammenlebende Mütter. Insgesamt beinhaltet unsere Stichprobe 1317 Beobachtungen. 
dells berechnet werden. Im Anschluss daran werden die monatlichen Übergänge aus Elternzeit in Erwerbstätigkeit geschätzt. ${ }^{8}$

Auf Basis der Einkommen aus der statischen Mikrosimulation werden die Erwerbsentscheidungen unter dem geltenden Recht 2006 und dem hypothetischen Szenario Elterngeld für 2006 vorausgesagt. Die Differenz zwischen diesen beiden Wahrscheinlichkeiten kann unter den Annahmen des mikroökonomischen Entscheidungsmodells als kausaler Effekt der Einführung des Elterngeldes interpretiert werden.

\subsection{Auswirkungen auf das Haushaltsnettoeinkommen}

Tabelle 1 stellt den Vergleich der monatlichen Haushaltsnettoeinkommen vor und nach der Einführung des Elterngeldes für verschiedene Charakteristika der Mutter oder des Haushaltes dar. Im Durchschnitt steigt das Einkommen bedingt durch das Elterngeld um rund 480 Euro. Eine Unterscheidung nach der Anzahl der Kinder zeigt, dass der größte Einkommensunterschied bei Familien mit einem Kind zu beobachten ist. Hier ist durch das Elterngeld das Haushaltsnettoeinkommen beinahe 600 Euro oder gut 27\% höher als im Falle des Erziehungsgeldes. Mütter mit einem Kind waren vor Geburt oft vollzeiterwerbstätig und erhalten deshalb ein relativ hohes Elterngeld. Hat die Mutter drei Kinder, so beträgt der Unterschied im Haushaltsnettoeinkommen nur noch etwa 220 Euro bzw. 7\%. Die Unterscheidung nach dem Bildungshintergrund der Mutter zeigt folgendes Ergebnis: Während der Anstieg im Haushaltsnettoeinkommen bei Müttern mit geringem Bildungsabschluss im ersten Lebensjahr des Kindes rund 430 Euro oder $18 \%$ beträgt, erreicht die Gruppe mit dem höchsten Bildungsabschluss ein Plus von 600 Euro oder rund $24 \%$. Die Unterschiede zwischen West- und Ostdeutschland liegen vor allem in den unterschiedlich hohen Einkommen begründet. In Westdeutschland nimmt das durchschnittliche Haushaltseinkommen um knapp 500 Euro (20\%) zu, während es mit etwa 400 Euro (19\%) in Ostdeutschland zwar absolut etwas weniger zunimmt, aber eine relativ ähnlich starke Zunahme aufweist. Mütter mit Migrationshintergrund profitieren bedingt durch eine durchschnittliche geringere Erwerbstätigkeit mit 400 Euro in geringerem Umfang als die Referenzgruppe. Insgesamt zeigt sich, dass vor allem das Haushaltseinkommen der Mütter steigt, die vor der Geburt eines Kindes erwerbstätig waren.

8 Eine detaillierte Darstellung des Arbeitsangebotsmodells findet sich in Wrohlich et al. (2012). 
Tabelle 1: Vergleich des verfügbaren Haushaltsnettoeinkommen vor und nach Einführung des Elterngeldes - erstes Jahr nach Geburt (alle Werte in Euro pro Monat)

\begin{tabular}{|c|c|c|c|}
\hline & $\begin{array}{c}<2007 \\
\text { (1) }\end{array}$ & $\begin{array}{c}>=2007 \\
(2)\end{array}$ & $\begin{array}{c}\text { Differenz } \\
(2)-(1)\end{array}$ \\
\hline Insgesamt & 2445 & 2924 & 479 \\
\hline \multicolumn{4}{|l|}{ Kinderzahl } \\
\hline 1 Kind & 2163 & 2754 & 591 \\
\hline 2 Kinder & 2607 & 2984 & 376 \\
\hline $3+$ Kinder & 3340 & 3566 & 226 \\
\hline \multicolumn{4}{|l|}{ Bildungsstand (Mutter) } \\
\hline Ohne Hochschulabschluss & 2421 & 2851 & 430 \\
\hline Mit Hochschulabschluss & 2534 & 3137 & 603 \\
\hline \multicolumn{4}{|l|}{ Region } \\
\hline Westdeutschland & 2518 & 3015 & 496 \\
\hline Ostdeutschland & 2103 & 2499 & 396 \\
\hline \multicolumn{4}{|l|}{ Haushaltsstruktur } \\
\hline Alleinerziehend & 1216 & 1687 & 471 \\
\hline Paarhaushalt & 2841 & 3322 & 481 \\
\hline \multicolumn{4}{|c|}{ Erwerbstätigkeit vor Geburt (Mutter) } \\
\hline Nein & 2244 & 2471 & 228 \\
\hline $\mathrm{Ja}$ & 2487 & 3019 & 532 \\
\hline \multicolumn{4}{|l|}{ Migrationshintergrund (Mutter) } \\
\hline Nein & 2519 & 3019 & 500 \\
\hline $\mathrm{Ja}$ & 2178 & 2582 & 404 \\
\hline
\end{tabular}

Quelle: SOEPv27 2005-2008 eigene Berechnungen, vgl. auch Wrohlich et al. (2012: Tabelle 2-3).

Neben der Betrachtung des durchschnittlichen Haushaltsnettoeinkommens, ist es auch interessant die Einkommensverteilung vor und nach Einführung des Elterngelds zu analysieren. Wie Tabelle 2 zeigt, sind nach Einführung des Elterngeldes alle Einkommenskategorien unterhalb von 3.000 Euro weniger stark besetzt als zuvor. Insbesondere in der Kategorie bis 1.000 Euro sinkt der Anteil der Haushalte von 8\% auf fast 2\%. Der Rückgang in den anderen Kategorien fällt weniger stark aus. Dafür steigen die entsprechenden Anteile in den höheren Einkommenskategorien. So betrachtet findet eine Verschiebung der Einkommensverteilung nach oben statt.

Tabelle 2: Anteile der Haushalte in Einkommensklassen vor und nach Einführung des Elterngeldes - erstes Jahr nach Geburt (in \%)

\begin{tabular}{lrr}
\hline $\begin{array}{l}\text { Einkommenskategorien } \\
\text { (Haushaltsnettoeinkommen in Euro pro Monat) }\end{array}$ & $\mathbf{2 0 0 7}$ & $\mathbf{> = 2 0 0 7}$ \\
\hline$<1.000$ & 8,2 & 1,9 \\
$1.001-1.500$ & 15,4 & 9,9 \\
$1.501-2.000$ & 16,1 & 15,7 \\
$2.001-2.500$ & 17,3 & 17,1 \\
$2.501-3.000$ & 14,9 & 15,5 \\
$3.001-3.500$ & 10,8 & 10,8 \\
$3.501-4.000$ & 9,3 & 9,0 \\
$4.001-4.500$ & 2,4 & 7,7 \\
$>4.501$ & 5,7 & 12,3 \\
\hline
\end{tabular}

Quelle: SOEPv27 2005-2008 eigene Berechnungen, vgl. auch Wrohlich et al. (2012: Tabelle 2-5). 


\subsection{Auswirkungen auf die Erwerbstätigkeit von Müttern}

In den folgenden Analysen wird der kausale Arbeitsangebotseffekt des Elterngeldes bezogen auf die mütterliche Erwerbsentscheidung identifiziert. Vorab kann festgehalten werden, dass die Veränderung der Erwerbsanreize des Elterngeldes entscheidend von zwei Faktoren abhängen: Zum einen vom Haushaltseinkommen, denn nur Familien mit niedrigem Haushaltseinkommen hatten Anspruch auf Erziehungsgeld. Zum zweiten vom Erwerbseinkommen vor Geburt des Kindes, denn dies hat wiederum Einfluss auf die Höhe des Elterngeldes. Tendenziell gilt: Für Mütter mit niedrigem Haushaltseinkommen gibt es im ersten Lebensjahr keine starke Änderung der Erwerbsanreize, dafür steigen für diese Gruppe die Erwerbsanreize im zweiten Lebensjahr aufgrund des Wegfalls des Erziehungsgeldes. Für Mütter mit einem hohen eigenen Erwerbseinkommen vor der Geburt und/oder hohem Haushaltseinkommen verändern sich die Erwerbsanreize im ersten Lebensjahr. Der Ausstieg aus der Erwerbstätigkeit wird durch das Elterngeld attraktiver, denn diese Mütter hätten kein Erziehungsgeld bekommen. Im zweiten Lebensjahr ändern sich für diese Mütter die Erwerbsanreize nicht - sie hätten auch vor der Einführung des Elterngeldes im zweiten Lebensjahr keine staatliche Leistung erhalten.

Die empirische Analyse zeigt die folgenden Ergebnisse: Im ersten Jahr nach der Geburt eines Kindes finden wir - mit Ausnahme der Gruppe der Mütter in Paarhaushalten mit zwei Kindern und niedrigen Einkommen - für alle Mütter in Paarhaushalten negative Beschäftigungseffekte durch die Einführung des Elterngeldes (Tabelle 3). Dies gilt sowohl für Teilzeit- als auch für Vollzeitbeschäftigung. Für Mütter in Paarhaushalten mit einem Kind ist die Wahrscheinlichkeit, dass sie in den ersten 12 Lebensmonaten ihres Kindes eine Teilzeitbeschäftigung aufnehmen, aufgrund des Elterngeldes um 1 Prozentpunkt niedriger. Die Wahrscheinlichkeit, eine Vollzeittätigkeit in diesem Zeitraum aufzunehmen, ist um 0,7 Prozentpunkte niedriger. Die stärksten Effekte finden wir für Mütter aus Paarhaushalten mit hohen Einkommen, hier sinkt die Übergangswahrscheinlichkeit in eine Teilzeiterwerbstätigkeit um 1,5 und in eine Vollzeiterwerbstätigkeit um 1 Prozentpunkt. Für Alleinerziehende finden wir im Durchschnitt keine Veränderungen der Erwerbstätigkeit im ersten Lebensjahr des Kindes aufgrund der Einführung des Elterngeldes.

Das Bild verändert sich jedoch während des zweiten Jahres nach der Geburt. Tabelle 4 zeigt die Wahrscheinlichkeit, dass Mütter im zweiten Jahr nach der Geburt eines Kindes eine Teilzeiterwerbstätigkeit aufnehmen. Hier finden wir für alle Frauen mit Partner positive Beschäftigungseffekte aufgrund der Einführung des Elterngeldes. So steigt z.B. die durchschnittliche Wahrscheinlichkeit für Mütter in Paarhaushalten mit einem Kind, in den Monaten 13 bis 24 nach der Geburt eine Teilzeiterwerbstätigkeit aufzunehmen, um 0,6 Prozentpunkte. Für Mütter in ostdeutschen Paarhaushalten steigt der Anteil sogar um über 2 Prozentpunkte. Für Mütter mit niedrigem Haushaltseinkommen ist der Effekt mit über 2,5 Prozentpunkten noch höher, aber auch in der Gruppe der Mütter mit hohen Einkommen finden wir signifikant positive Effekte. Für Alleinerziehende finden wir negative Effekte in Bezug auf die Wahrscheinlichkeit, im zweiten Lebensjahr des Kindes eine Teilzeittätigkeit aufzunehmen. Für diese Gruppe sind jedoch positive Effekte in Bezug auf Vollzeitbeschäftigung festzumachen (vgl. Tabelle 4). Die Wahrscheinlichkeit, dass eine alleinerziehende Mutter mit einem Kind im zweiten Jahr nach der Geburt eine Vollzeittätigkeit aufnimmt, hat sich im Westen um 0,5 Prozentpunkte und im Osten um über 1 Pro- 
zentpunkt erhöht. Für Mütter mit Partner finden wir tendenziell niedrigere Effekte in Bezug auf die Vollzeiterwerbstätigkeit. Den höchsten Effekt finden wir auch hier für Mütter in Ostdeutschland: Für diese Mütter mit einem Kind erhöht sich die Wahrscheinlichkeit, im zweiten Jahr nach der Geburt eine Vollzeittätigkeit aufzunehmen um 1,3 Prozentpunkte, für Mütter mit zwei Kindern um 0,9 Prozentpunkte.

Tabelle 3: Veränderung der Übergangswahrscheinlichkeit in Teilzeit- oder Vollzeiterwerbstätigkeit im ersten Jahr nach der Geburt aufgrund des Elterngeldes $(>=2007)$

\begin{tabular}{|c|c|c|c|c|c|}
\hline & & $\begin{array}{l}\text { Übergangswahr- } \\
\text { scheinlichkeit in } \\
\text { Teilzeiterwerbs- } \\
\text { tätigkeit }<2007 \\
\text { (in Prozent) }\end{array}$ & $\begin{array}{l}\text { Veränderung der } \\
\text { Übergangswahr- } \\
\text { scheinlichkeit in } \\
\text { Teilzeiterwerbs- } \\
\text { tätigkeit >= 2007 } \\
\text { (in Prozent- } \\
\text { punkten) }\end{array}$ & $\begin{array}{l}\text { Übergangswahr- } \\
\text { scheinlichkeit in } \\
\text { Vollzeiterwerbs- } \\
\text { tätigkeit < } 2007 \\
\text { (in Prozent) }\end{array}$ & $\begin{array}{l}\text { Veränderung der } \\
\text { Übergangswahr- } \\
\text { scheinlichkeit in } \\
\text { Vollzeiterwerbs- } \\
\text { tätigkeit >= 2007 } \\
\text { (in Prozent- } \\
\text { punkten) }\end{array}$ \\
\hline \multirow[t]{5}{*}{$\begin{array}{l}\text { Frauen in Paarhaushalten } \\
\text { mit einem Kind }\end{array}$} & Gesamt & 22,0 & $\begin{array}{c}-1,05 \\
(-1,74--0,36)\end{array}$ & 4,3 & $\begin{array}{c}-0,69 \\
(-1,26--0,12)\end{array}$ \\
\hline & West & 22,4 & $\begin{array}{c}-1,12 \\
(-1,85--0,40\end{array}$ & 3,6 & $\begin{array}{c}-\mathbf{0 , 5 9} \\
(-1,08--0,10)\end{array}$ \\
\hline & Ost & 20,0 & $\begin{array}{c}-\mathbf{0 , 5 1} \\
(-0,88--0,14)\end{array}$ & 10,5 & $\begin{array}{c}-1,01 \\
(-1,85--0,17)\end{array}$ \\
\hline & $\begin{array}{l}\text { Niedriges } \\
\text { Einkommen }\end{array}$ & 21,0 & $\begin{array}{c}-\mathbf{0 , 4 4} \\
(-0,73--0,15)\end{array}$ & 3,8 & $\begin{array}{c}-\mathbf{0 , 3 5} \\
(-0,62--0,08)\end{array}$ \\
\hline & $\begin{array}{l}\text { Hohes } \\
\text { Einkommen }\end{array}$ & 22,5 & $\begin{array}{c}-1,5 \\
(-2,48--0,52)\end{array}$ & 4,6 & $\begin{array}{c}-0,81 \\
(-1,48--0,13)\end{array}$ \\
\hline \multirow[t]{5}{*}{$\begin{array}{l}\text { Frauen in Paarhaushalten } \\
\text { mit } 2 \text { Kindern }\end{array}$} & Gesamt & 20,3 & $\begin{array}{c}-\mathbf{0 , 8 3} \\
(-1,38--0,28)\end{array}$ & 2,7 & $\begin{array}{c}-\mathbf{0 , 3 0} \\
(-0,55--0,05)\end{array}$ \\
\hline & West & 21,0 & $\begin{array}{c}-\mathbf{0 , 8 4} \\
(-1,41--0,27)\end{array}$ & 2,3 & $\begin{array}{c}-\mathbf{0 , 2 5} \\
(-0,47--0,03)\end{array}$ \\
\hline & Ost & 18,1 & $\begin{array}{c}-\mathbf{0 , 2 8} \\
(-0,48-0,08)\end{array}$ & 6,4 & $\begin{array}{c}-0,45 \\
(-0,82--0,08)\end{array}$ \\
\hline & $\begin{array}{l}\text { Niedriges } \\
\text { Einkommen }\end{array}$ & 18,1 & $\begin{array}{c}\mathbf{0 , 3 2} \\
(0,12-0,52)\end{array}$ & 2,2 & $\begin{array}{c}-\mathbf{0 , 0 6} \\
(-0,10--0,02)\end{array}$ \\
\hline & $\begin{array}{l}\text { Hohes } \\
\text { Einkommen }\end{array}$ & 20,4 & $\begin{array}{c}-\mathbf{0 , 6 3} \\
(-1,05--0,21)\end{array}$ & 2,9 & $\begin{array}{c}-\mathbf{0 , 4 2} \\
(-0,78--0,06)\end{array}$ \\
\hline \multirow[t]{3}{*}{$\begin{array}{l}\text { Alleinerziehende mit einem } \\
\text { Kind }\end{array}$} & Gesamt & 16,8 & 0,0 & 3,5 & 0,00 \\
\hline & West & 17,1 & 0,0 & 2,9 & 0,00 \\
\hline & Ost & 15,9 & 0,0 & 8,7 & 0,00 \\
\hline
\end{tabular}

Fettgedruckte Werte zeigen an, dass sie statistisch signifikant sind.

Werte in Klammern geben 95\%-Konfidenzintervalle an.

Quelle: Eigene Schätzungen auf Basis des SOEPv27 2005-2008, vgl. auch Wrohlich et al. (2012: Tabellen 7-18 und 7-19).

Das Ergebnis einer Erhöhung der Teilzeiterwerbstätigkeit von Müttern mit niedrigem Einkommen im zweiten Lebensjahr des Kindes um 2 Prozentpunkte kann im Vergleich zu anderen tatsächlichen oder diskutierten Reformen im Bereich der Familien- und Sozialpo- 
litik als relativ großer Effekt beschrieben werden. Studien, die auf ähnlichen Methoden basieren und z.B. Reformen im Bereich der Kindertagesbetreuung untersuchen (Abschaffung aller Elternbeiträge für Kindertagesbetreuungseinrichtungen oder Ausbau der Betreuungsplätze für alle Kinder unter drei Jahren) finden für solche Reformen Steigerungen der Partizipationsquote von Müttern mit Kindern unter drei Jahren in ähnlicher Größenordnung (vgl. dazu Wrohlich 2011). Auch die sehr radikale hypothetische Reform einer vollständigen Abschaffung des Ehegattensplittings zugunsten einer reinen Individualbesteuerung hätte für den Durchschnitt aller verheirateten Frauen nur unwesentlich höhere Partizipationseffekte zur Folge (vgl. dazu Bach et al. 2011).

Tabelle 4: Veränderung der Übergangswahrscheinlichkeit in Vollzeit- oder Teilzeiterwerbstätigkeit im zweiten Jahr nach der Geburt aufgrund des Elterngeldes $(>=2007)$

\begin{tabular}{|c|c|c|c|c|c|}
\hline & & $\begin{array}{l}\text { Übergangswahr- } \\
\text { scheinlichkeit in } \\
\text { Teilzeiterwerbstä- } \\
\text { tigkeit < 2007 } \\
\text { (in Prozent) }\end{array}$ & $\begin{array}{l}\text { Veränderung der } \\
\text { Übergangswahr- } \\
\text { scheinlichkeit in } \\
\text { Teilzeiterwerbs- } \\
\text { tätigkeit }>=2007 \\
\text { (in Prozent- } \\
\text { punkten) }\end{array}$ & $\begin{array}{l}\text { Übergangswahr- } \\
\text { scheinlichkeit in } \\
\text { Vollzeiterwerbs- } \\
\text { tätigkeit < 2007 } \\
\text { (in Prozent) }\end{array}$ & $\begin{array}{l}\text { Veränderung der } \\
\text { Übergangswahr- } \\
\text { scheinlichkeit in } \\
\text { Vollzeiterwerbstä- } \\
\text { tigkeit }>=2007 \\
\text { (in Prozent- } \\
\text { punkten) }\end{array}$ \\
\hline \multirow[t]{5}{*}{$\begin{array}{l}\text { Frauen in Paarhaus- } \\
\text { halten mit einem Kind }\end{array}$} & Gesamt & 22,9 & $\begin{array}{c}\mathbf{0 , 5 6} \\
(0,11-1,01)\end{array}$ & 4,9 & $\begin{array}{c}\mathbf{0 , 1 2} \\
(0,002-0,24)\end{array}$ \\
\hline & West & 23,4 & $\begin{array}{c}\mathbf{0 , 5 6} \\
(0,09-1,03)\end{array}$ & 4,1 & $\begin{array}{c}\mathbf{0 , 1 0} \\
(0,002-0,20)\end{array}$ \\
\hline & Ost & 17,4 & $\begin{array}{c}2, \mathbf{1 3} \\
(0,78-3,48)\end{array}$ & 10,3 & $\begin{array}{c}\mathbf{1 , 2 8} \\
(0,14-2,42)\end{array}$ \\
\hline & $\begin{array}{l}\text { Niedriges } \\
\text { Einkommen }\end{array}$ & 19,6 & $\begin{array}{c}2,56 \\
(1,09-4,03)\end{array}$ & 4,1 & $\begin{array}{c}\mathbf{0 , 5 4} \\
(0,05-1,03)\end{array}$ \\
\hline & $\begin{array}{l}\text { Hohes } \\
\text { Einkommen }\end{array}$ & 23,1 & $\begin{array}{c}\mathbf{0 , 7 6} \\
(0,12-1,39)\end{array}$ & 5,1 & $\begin{array}{c}\mathbf{0 , 1 6} \\
(0,00-0,32)\end{array}$ \\
\hline \multirow[t]{5}{*}{$\begin{array}{l}\text { Frauen in Paarhaus- } \\
\text { halten mit } 2 \text { Kindern }\end{array}$} & Gesamt & 21,2 & $\begin{array}{c}1,16 \\
(0,42-1,91)\end{array}$ & 3,1 & $\begin{array}{c}\mathbf{0 , 1 7} \\
(0,01-0,33)\end{array}$ \\
\hline & West & 21,9 & $\begin{array}{c}\mathbf{0 , 7 1} \\
(0,22-1,20)\end{array}$ & 2,6 & $\begin{array}{c}\mathbf{0 , 0 8} \\
(0,002-0,16)\end{array}$ \\
\hline & Ost & 17,1 & $\begin{array}{c}1,66 \\
(0,64-2,68)\end{array}$ & 6,7 & $\begin{array}{c}\mathbf{0 , 9 1} \\
(0,09-1,73)\end{array}$ \\
\hline & $\begin{array}{l}\text { Niedriges } \\
\text { Einkommen }\end{array}$ & 19,5 & $\begin{array}{c}1,20 \\
(0,51-1,89)\end{array}$ & 2,5 & $\begin{array}{c}\mathbf{0 , 3 3} \\
(0,04-0,62)\end{array}$ \\
\hline & $\begin{array}{l}\text { Hohes } \\
\text { Einkommen }\end{array}$ & 22,4 & $\begin{array}{c}\mathbf{0 , 3 2} \\
(0,05-0,59)\end{array}$ & 3,3 & $\begin{array}{c}\mathbf{0 , 0 5} \\
(0,00-0,09)\end{array}$ \\
\hline \multirow[t]{3}{*}{$\begin{array}{l}\text { Alleinerziehende mit } \\
\text { einem Kind }\end{array}$} & Gesamt & 18,7 & $\begin{array}{c}-\mathbf{0 , 0 9} \\
(-0,17--0,01)\end{array}$ & 3,8 & $\begin{array}{c}\mathbf{0 , 5 6} \\
(0,03-1,10)\end{array}$ \\
\hline & West & 19,1 & $\begin{array}{c}-0,07 \\
(-0,15-0,01)\end{array}$ & 3,2 & $\begin{array}{c}\mathbf{0 , 4 8} \\
(0,03-0,93)\end{array}$ \\
\hline & Ost & 16,4 & $\begin{array}{c}-0,20 \\
(-0,40--0,004) \\
\end{array}$ & 9,3 & $\begin{array}{c}1,27 \\
(0,17-2,37) \\
\end{array}$ \\
\hline
\end{tabular}

Fettgedruckte Werte zeigen an, dass sie statistisch signifikant sind. Werte in Klammern geben 95\%-Konfidenzintervalle an.

Quelle: Eigene Schätzungen auf Basis des SOEPv27 2005-2008, vgl. auch Wrohlich et al. (2012: Tabellen 3-8 und 3-9). 
Tabelle 5: Veränderung der Übergangswahrscheinlichkeit in Teilzeit- oder Vollzeiterwerbstätigkeit im ersten und zweiten Jahr nach der Geburt aufgrund des Elterngeldes $(>=2007)$ (Gesamteffekt)

\begin{tabular}{|c|c|c|c|c|c|}
\hline & & $\begin{array}{l}\text { Übergangswahr- } \\
\text { scheinlichkeit in } \\
\text { Teilzeiterwerbs- } \\
\text { tätigkeit } \\
<2007 \text { (in Prozent) }\end{array}$ & $\begin{array}{c}\text { Veränderung der } \\
\text { Übergangswahr- } \\
\text { scheinlichkeit in } \\
\text { Teilzeiterwerbs- } \\
\text { tätigkeit } \\
>>=2007 \text { (in Pro- } \\
\text { zentpunkten) }\end{array}$ & $\begin{array}{c}\text { Übergangswahr- } \\
\text { scheinlichkeit in } \\
\text { Vollzeiterwerbs- } \\
\text { tätigkeit } \\
<2007 \text { (in Prozent) }\end{array}$ & $\begin{array}{l}\text { Veränderung der } \\
\text { Übergangswahr- } \\
\text { scheinlichkeit in } \\
\text { Vollzeiterwerbs- } \\
\text { tätigkeit } \\
\text { >= } 2007 \text { (in Pro- } \\
\text { zentpunkten) }\end{array}$ \\
\hline \multirow[t]{5}{*}{$\begin{array}{l}\text { Frauen in Paarhaus- } \\
\text { halten mit einem Kind }\end{array}$} & Gesamt & 44,8 & $\begin{array}{c}-\mathbf{0 , 4 9} \\
(-0,84--0,14)\end{array}$ & 9,2 & $\begin{array}{c}-\mathbf{0 , 5 8} \\
(-1,09--0,07)\end{array}$ \\
\hline & West & 45,8 & $\begin{array}{c}-\mathbf{0 , 5 6} \\
(-0,93--0,19)\end{array}$ & 7,8 & $\begin{array}{c}-\mathbf{0 , 4 9} \\
(-0,92--0,06)\end{array}$ \\
\hline & Ost & 37,3 & $\begin{array}{c}1,62 \\
(0,58-2,66)\end{array}$ & 20,9 & $\begin{array}{c}0,28 \\
(-0,54-1,10)\end{array}$ \\
\hline & $\begin{array}{l}\text { Niedriges } \\
\text { Einkommen }\end{array}$ & 40,6 & $\begin{array}{c}\mathbf{2 , 1 3} \\
(0,90-3,37)\end{array}$ & 7,9 & $\begin{array}{c}0,19 \\
(-0,20-0,58)\end{array}$ \\
\hline & $\begin{array}{l}\text { Hohes Ein- } \\
\text { kommen }\end{array}$ & 45,6 & $\begin{array}{c}-\mathbf{0 , 7 4} \\
(-1,24--0,24)\end{array}$ & 9,6 & $\begin{array}{c}-\mathbf{0 , 6 5} \\
(-1,23--0,06)\end{array}$ \\
\hline \multirow[t]{5}{*}{$\begin{array}{l}\text { Frauen in Paarhaus- } \\
\text { halten mit } 2 \text { Kindern }\end{array}$} & Gesamt & 41,5 & $\begin{array}{c}\mathbf{0 , 3 3} \\
(0,04-0,62)\end{array}$ & 5,8 & $\begin{array}{c}-0,13 \\
(-0,33-0,07)\end{array}$ \\
\hline & West & 42,9 & $\begin{array}{c}-0,13 \\
(-0,31-0,05)\end{array}$ & 4,9 & $\begin{array}{c}-0,17 \\
(-0,35-0,01)\end{array}$ \\
\hline & Ost & 35,1 & $\begin{array}{c}1,38 \\
(0,54-2,22)\end{array}$ & 13,1 & $\begin{array}{c}0,46 \\
(-0,19-1,11)\end{array}$ \\
\hline & $\begin{array}{l}\text { Niedriges } \\
\text { Einkommen }\end{array}$ & 37,6 & $\begin{array}{c}1,52 \\
(0,66-2,38)\end{array}$ & 4,7 & $\begin{array}{c}\mathbf{0 , 2 8} \\
(0,01-0,55)\end{array}$ \\
\hline & $\begin{array}{l}\text { Hohes Ein- } \\
\text { kommen }\end{array}$ & 42,8 & $\begin{array}{c}-0,31 \\
(-0,53--0,09)\end{array}$ & 6,2 & $\begin{array}{c}-0,37 \\
(-0,70--0,05)\end{array}$ \\
\hline \multirow[t]{3}{*}{$\begin{array}{l}\text { Alleinerziehende mit } \\
\text { einem Kind }\end{array}$} & Gesamt & 35,6 & $\begin{array}{c}-\mathbf{0 , 0 9} \\
(-0,17--0,01)\end{array}$ & 7,3 & $\begin{array}{c}\mathbf{0 , 5 6} \\
(0,31-1,09)\end{array}$ \\
\hline & West & 36,2 & $\begin{array}{c}-\mathbf{0 , 0 7} \\
(-0,15-0,01)\end{array}$ & 6,2 & $\begin{array}{c}\mathbf{0 , 4 8} \\
(0,03-0,93)\end{array}$ \\
\hline & Ost & 32,3 & $\begin{array}{c}-\mathbf{0 , 2 0} \\
(-0,40--0,004)\end{array}$ & 18,0 & $\begin{array}{c}1,27 \\
(0,17-2,37) \\
\end{array}$ \\
\hline
\end{tabular}

Fettgedruckte Werte zeigen an, dass sie statistisch signifikant sind.

Werte in Klammern geben 95\%-Konfidenzintervalle an.

Quelle: Eigene Schätzungen auf Basis des SOEPv27 2005-2008, vgl. auch Wrohlich et al. (2012: Tabellen 7-20 und 7-21).

Die Gesamteffekte für Teilzeit- und Vollzeitbeschäftigung, also die Wahrscheinlichkeit, irgendwann innerhalb der ersten zwei Lebensjahre eine Erwerbstätigkeit aufzunehmen, sind in Tabelle 5 dargestellt. Der Gesamteffekt über die ersten zwei Lebensjahre fällt je nach Gruppen unterschiedlich aus. Für Mütter in Ostdeutschland und für Mütter in Paarhaushalten mit niedrigen Einkommen in Ost- und Westdeutschland finden wir signifikant positive Effekte. Da alleinerziehende Frauen ihr Arbeitsangebot im ersten Jahr nach der Geburt durch die Einführung des Elterngeldes nicht verändern, entspricht der Gesamteffekt dem Beschäftigungseffekt im zweiten Jahr. Wir finden negative Beschäftigungseffekte in Teilzeitbeschäftigung, die von positiven Effekten in Vollzeitarbeit kompensiert 
werden. Der Vollzeiteffekt ist mit 1,3 Prozentpunkten besonders groß für Alleinerziehende in Ostdeutschland. Vollzeiterwerbstätigkeit ist für diese Gruppe von Müttern daher durch die Elterngeldreform deutlich attraktiver geworden.

Tabelle 6: Durchschnittliche Dauer der Erwerbsunterbrechung in den ersten zwei Jahren nach der Geburt in Monaten

\begin{tabular}{|c|c|c|c|c|}
\hline & & $\begin{array}{c}<2007 \\
(1)\end{array}$ & $\begin{array}{c}>=2007 \\
(2)\end{array}$ & $\begin{array}{l}\text { Differenz } \\
\text { (2)-(1) }\end{array}$ \\
\hline \multirow[t]{5}{*}{ Paarhaushalt mit einem Kind } & Gesamt & 17,05 & 17,32 & $\begin{array}{c}\mathbf{0 , 2 7} \\
(0,11-0,43)\end{array}$ \\
\hline & West & 17,13 & 17,40 & $\begin{array}{c}\mathbf{0 , 2 7} \\
(0,11-0,43)\end{array}$ \\
\hline & Ost & 16,24 & 16,24 & $\begin{array}{c}-0,01 \\
(-0,11--0,02)\end{array}$ \\
\hline & $\begin{array}{l}\text { Niedriges } \\
\text { Einkommen }\end{array}$ & 17,67 & 17,56 & $\begin{array}{c}-\mathbf{0 , 1 0} \\
(-0,18--0,02)\end{array}$ \\
\hline & $\begin{array}{l}\text { Hohes } \\
\text { Einkommen }\end{array}$ & 16,87 & 17,23 & $\begin{array}{c}\mathbf{0 , 3 6} \\
(0,14-0,58)\end{array}$ \\
\hline \multirow[t]{5}{*}{ Paarhaushalt mit 2 Kindern } & Gesamt & 17,96 & 18,06 & $\begin{array}{c}\mathbf{0 , 1 0} \\
(0,02-0,18)\end{array}$ \\
\hline & West & 17,89 & 18,03 & $\begin{array}{c}\mathbf{0 , 1 4} \\
(0,06-0,22)\end{array}$ \\
\hline & Ost & 17,72 & 17,64 & $\begin{array}{c}\mathbf{- 0 , 0 7} \\
(-0,13--0,01)\end{array}$ \\
\hline & $\begin{array}{l}\text { Niedriges } \\
\text { Einkommen }\end{array}$ & 18,64 & 18,47 & $\begin{array}{c}-\mathbf{0 , 1 7} \\
(-0,27--0,07)\end{array}$ \\
\hline & $\begin{array}{l}\text { Hohes } \\
\text { Einkommen }\end{array}$ & 17,78 & 17,95 & $\begin{array}{c}\mathbf{0 , 1 7} \\
(0,07-0,27)\end{array}$ \\
\hline \multirow[t]{5}{*}{$\begin{array}{l}\text { Alleinerziehende } \\
\text { mit einem Kind }\end{array}$} & Gesamt & 18,59 & 18,55 & $\begin{array}{c}-\mathbf{0 , 0 4} \\
(-0,08--0,001)\end{array}$ \\
\hline & West & 18,67 & 18,64 & $\begin{array}{c}-0,03 \\
(-0,07-0,01)\end{array}$ \\
\hline & Ost & 17,53 & 17,43 & $\begin{array}{c}\mathbf{- 0 , 0 9} \\
(-0,17--0,01)\end{array}$ \\
\hline & $\begin{array}{l}\text { Niedriges } \\
\text { Einkommen }\end{array}$ & 18,71 & 18,67 & $\begin{array}{c}-\mathbf{0 , 0 4} \\
(-0,08--0,001)\end{array}$ \\
\hline & $\begin{array}{l}\text { Hohes } \\
\text { Einkommen }\end{array}$ & 18,5 & 18,45 & $\begin{array}{c}\mathbf{0 , 0 4} \\
(0,001-0,08)\end{array}$ \\
\hline
\end{tabular}

Fettgedruckte Werte zeigen an, dass sie statistisch signifikant sind.

Werte in Klammern geben 95\%-Konfidenzintervalle an.

Quelle: Eigene Schätzungen auf Basis des SOEPv27 2005-2008, vgl. auch Wrohlich et al. (2012: Tabelle 3-10).

Die Ergebnisse bezüglich der Übergänge in Erwerbstätigkeit können auch als Dauer der Erwerbsunterbrechungen dargestellt werden. Tabelle 6 fasst die Veränderungen in der Dauer der Erwerbsunterbrechungen zusammen. In den ersten beiden Spalten von Tabelle 6 wird die durchschnittliche Dauer in Nichtbeschäftigung während der ersten 24 Monate nach der Geburt für die Jahre vor 2007 und ab 2007 ausgewiesen. In der letzten Spalte berechnen wir die Differenz zwischen den beiden Perioden. Die durchschnittliche Erwerbs- 
unterbrechung für Mütter in Paarhaushalten mit einem oder zwei Kindern hat sich durch das Elterngeld leicht erhöht (plus 0,3 Monate). Für Mütter mit niedrigem Haushaltseinkommen hat sich die durchschnittliche Erwerbsunterbrechung jedoch verkürzt, und zwar um 0,1 Monate bei Müttern in Paarhaushalten mit 1 Kind und um fast 0,2 Monate bei Müttern mit zwei Kindern. Bei alleinerziehenden Müttern hat sich auch im Gesamtdurchschnitt die durchschnittliche Erwerbsunterbrechung durch die Einführung des Elterngeldes leicht verkürzt (0,04 Monate). Wie bereits oben erläutert, spiegeln diese Effekte die Anreizwirkungen wider: Für Mütter mit hohem Einkommen, die keinen Anspruch auf Erziehungsgeld gehabt hätten, aber Anspruch auf hohes Elterngeld haben, ist es attraktiver geworden, im ersten Lebensjahr des Kindes die Erwerbstätigkeit zu unterbrechen. Im zweiten Lebensjahr des Kindes haben sich für diese Gruppe von Müttern die Anreize durch das Elterngeld nicht verändert: Vorher wie nachher erhält diese Mutter im zweiten Lebensjahr keine staatliche Leistung mehr. Das ist der Grund dafür, dass wir z.B. für Mütter mit einem Kind in Westdeutschland eine Verlängerung der Erwerbsunterbrechung finden. Für Mütter mit niedrigem Haushaltseinkommen und niedrigem Elterngeldanspruch hingegen haben sich die Erwerbsanreize im ersten Jahr nicht besonders stark verändert, dafür ist im zweiten Lebensjahr eine Erwerbstätigkeit attraktiver geworden. So lässt sich erklären, warum wir z.B. für Mütter in Paarhaushalten mit zwei Kindern und niedrigem Einkommen eine Verkürzung der Erwerbsdauer finden.

\section{Schlussbemerkungen}

Das Ziel des vorliegenden Beitrags war es den Effekt des Elterngeldes auf das Haushaltseinkommen von jungen Familien und das Arbeitsangebot von Müttern zu schätzen. Die Ergebnisse haben gezeigt, dass das Haushaltseinkommen von Familien im ersten Lebensjahr im Durchschnitt aller Familien durch das Elterngeld ansteigt. Dies ist insbesondere für mittlere und höhere Einkommen der Fall. Dies entspricht dem Ziel der Reform den Einkommensverlust nach der Geburt zu glätten und für das erste Jahr einen sogenannten „Schonraum“ zu gewährleisten. Der Übergang von einem bedarfsgeprüften Transfer zu der neuen einkommensabhängigen Lohnersatzleistung führt gleichzeitig dazu, dass höhere Einkommen relativ stärker vom Elterngeld profitieren als untere Einkommensgruppen. ${ }^{9}$ Dieser Anstieg der Ungleichheit war Teil des Paradigmenwechsels der mit dem Elterngeld in der deutschen Familienpolitik eingeleitet wurde. Einschränkend muss aber hinzugefügt werden, dass die Leistungen des Elterngeldes nachträglich insbesondere für Geringverdiener erhöht wurden, sodass die Nettoersatzrate bei geringen Einkommen heute sogar $100 \%$ beträgt.

Im zweiten Lebensjahr verlieren vor allem einkommensschwächere Familien die Transferleistung des früheren Erziehungsgeldes. Auch hier könnte man argumentieren, dass die Ungleichheit durch die Reform zunimmt. Allerdings steigen dadurch ebenfalls die Erwerbsanreize für diese Gruppe und wenigstens langfristig könnte die Ungleichheit wieder sinken. Im Einklang mit den veränderten Arbeitsanreizen zeigt unsere empirische Analyse, dass die Erwerbstätigkeit von Müttern im Mittel im ersten Lebensjahr zurück-

9 Für eine kritische Analyse von Verteilungswirkungen des Elterngeldes vgl. Henninger et al. (2008). 
geht und im zweiten Lebensjahr zunimmt. Die positiven Beschäftigungseffekte im zweiten Lebensjahr des Kindes entsprechen den gewollten Anreizeffekten der Einführung des Elterngeldes. Vor allem für Haushalte, die früher Erziehungsgeld bekommen hätten, steigen im zweiten Jahr die Erwerbsanreize - deshalb finden wir für diese Gruppe die höchsten Effekte. Die höheren Effekte in Ostdeutschland haben vermutlich zwei Ursachen. Erstens sind dort die Einkommen im Durchschnitt niedriger als in Westdeutschland und zweitens ist die Kinderbetreuungsinfrastruktur besser ausgebaut. Es ist anzunehmen, dass die Reform komplementär zu einem weiteren Ausbau der Kindertagesbetreuung - insbesondere für Kinder unter drei Jahren - ist und auch die Erwerbstätigkeit in Westdeutschland im zweiten Lebensjahr des Kindes noch weiter ansteigen kann. Allerdings kann es hier aufgrund des Einkommenseffektes des 2013 beschlossenen Betreuungsgeldes auch dazu kommen, dass insbesondere untere Einkommensgruppen in geringerem Umfang Erwerbsarbeit aufnehmen. Hier zeigt sich, dass es zwar eine starke Bewegung in der Politik zur Verbesserung der Vereinbarkeit von Familie und Beruf gibt, dass dieses familienpolitische Ziel aber keineswegs ausschließlich gilt.

Mit der Zunahme einer Erwerbstätigkeit im zweiten Lebensjahr, wie sie durch das Elterngeld bedingt wird, kehren Mütter früher aus der Elternzeit zurück bzw. nutzen die Möglichkeiten einer Teilzeittätigkeit während der Elternzeit. Diese frühere Rückkehr könnte einem weiteren Ziel der nachhaltigen Familienpolitik, dem einer frühen Förderung von Kindern (vgl. Ristau 2006) entgegenstehen, wenn davon ausgegangen wird, dass längere Elternzeiten für die frühe Förderung von Kindern von Bedeutung sind. Einschlägige bildungs- und familienökonomische Analysen können dafür allerdings keine bzw. keine eindeutigen Hinweise finden. Darüber hinaus geben deskriptive Auswertungen von Wrohlich et al. (2012) auch Hinweise darauf, dass seit dem Elterngeld Väter im Mittel mehr Zeit mit Aufgaben der Kinderbetreuung verbringen - von Müttern wird diese Zeit häufig für den (Wieder-)Einstieg in das Erwerbsleben genutzt. Ein früherer Erwerbseinstieg von Müttern, wie er durch die Reform des Elterngeldes bedingt ist, kann insbesondere im unteren Einkommensbereich sehr viel eher dazu beitragen, dass Mütter eigenständig ihre wirtschaftliche Existenz sichern können. Ökonomische Untersuchungen geben vielfältige Hinweise darauf, wie wichtig kürzere Erwerbsunterbrechungen für mittel- und langfristige Lohnentwicklungen sind (vgl. z.B. Boll 2011 oder Geyer/Steiner 2007).

Insgesamt erreicht das Elterngeld die hier untersuchten Ziele. Eine Abschaffung desselben oder auch eine Reduktion der Leistungshöhe wie sie von einigen wenigen andiskutiert wurde, wäre nicht nur in Hinblick auf eine verlässliche Familienpolitik kontraproduktiv, sondern würde auch die wirtschaftliche Stabilität von Familien in den ersten Lebensjahren von Kindern beeinträchtigen.

Unsere Studie kann auf Basis eines einfachen ökonomischen Modells den kausalen Effekt des Elterngeldes auf das Arbeitsangebot ableiten. Einschränkend muss aber betont werden, dass wir in diesem Modell keine Interaktionen mit anderen familienpolitischen Maßnahmen explizit abbilden. Die Studie ist auch insofern beschränkt, da sie zum einen nur kurzfristige Effekte betrachtet und primär finanzielle Anreize einbezieht. Subjektive Faktoren, wie Einstellungen und Normen sind für die Entscheidung von Eltern jedoch auch von großer Bedeutung - sie werden hier jedoch nicht adressiert. Hierzu sind primär soziologische Ansätze notwendig. Auch andere geschlechterbezogene Überlegungen oder politikwissenschaftliche Ansätze, welche eine Einordnung des Elterngeldes in familienpo- 
litische Diskurse ergeben, werden hier nicht diskutiert (vgl. dazu z.B. Wimbauer/Henninger 2008). Vielmehr fokussiert diese Studie auf eine empirische Wirkungsanalyse auf der Basis von Mikrodaten.

\section{Danksagung}

Wir danken Martin Bujard und zwei anonymen Gutachtern für hilfreiche Anmerkungen.

\section{Literatur}

Bach, S., Geyer, J., Haan, P. \& Wrohlich K. (2011). Reform des Ehegattensplittings: Nur eine reine Individualbesteuerung erhöht die Erwerbsanreize deutlich. DIW Wochenbericht, 41, S. 13-19.

Bach, S., Geyer, J., Haan, P. \& Wrohlich K. (2012). Schwache Argumente für das Ehegattensplitting. Wirtschaftsdienst, 9, S. 620-62.

Baker, M. \& Milligan, K. (2008). Evidence from maternity leave expansions of the impact of maternal care on early childhood development. Journal of Human Resources, 45, 1, S. 1-32.

Berger, L. M. \& Waldvogel, J. (2004). Maternity leave and the employment of new mothers in the United States, Journal of Population Economics, 17, 2, S. 331-349.

Boll, C. (2011). Lohneinbußen von Frauen durch geburtsbedingte Erwerbsunterbrechungen. Der Schattenpreis von Kindern und dessen mögliche Auswirkungen auf weibliche Spezialisierungsentscheidungen im Haushaltszusammenhang. Eine quantitative Analyse auf Basis von SOEP-Daten. Frankfurt am Main et al.: Verlag Peter Lang (Monografische Dissertationsschrift, Reihe „Sozialökonomische Schriften“"(Hrsg.: B. Rürup \& W. Sesselmeier)).

Bujard, M., Dorbritz, J., Grünheid, E., Kühntopf, S., Lück, D., Naderi, R., Passet, J. \& Ruckdeschel, K. (2012) (Hrsg.). (Keine) Lust auf Kinder ? - Geburtenentwicklung in Deutschland. Wiesbaden: Bundesinstitut für Bevölkerungsforschung.

Bundesministerium für Familie, Senioren, Frauen und Jugend (BMFSFJ) (Hrsg.) (2012). Elterngeldmonitor. Berlin: Bundesministerium für Familie, Senioren, Frauen und Jugend.

Deutscher Bundestag (2006). Entwurf eines Gesetzes zur Einführung des Elterngeldes. Berlin: Deutscher Bundestag (Drucksache 16/1889 vom 20. Juni 2006).

Dustmann, C. \& Schönberg; U. (2012). Expansions in maternity leave coverage and children's long-term outcomes. American Economic Journal: Applied Economics, 4, 3, S. 190-224.

Duvander, A.-Z. \& Andersson, G. (2006). Gender equality and fertility in Sweden: A study on the impact of the father's uptake of parental leave on continued childbearing. Marriage \& Family Review, 39, 1-2, S. 121-142.

Ekberg, J., Eriksson, R. \& Friebel, G. (2013). Parental leave: A policy evaluation of the Swedish "daddymonth reform", Journal of Public Economics, 97, S. 131-143.

Geisler, E. \& Kreyenfeld, M. (2011). Against all odds: Fathers' use of parental leave in Germany. Journal of European Social Policy, 21, 1, S. 88-99.

Geyer, J. \& Steiner, V. (2007). Short-run and long-term effects of childbirth on mothers' employment and working hours across institutional regimes: An empirical analysis based on the European Community Household Panel. Bonn: Forschungsinstitut für die Zukunft der Arbeit (IZA Discussion Paper Nr. 2693).

Geyer, J., Haan, P., Spieß, C. K. \& Wrohlich, K. (2012). Elterngeld: Mütter kehren früher in den Beruf zurück. DIW Wochenbericht, 9, S. 3-10.

Greene, William H. (2003): Econometric analysis. Upper Saddle River, NJ: Prentice Hall (5. Auflage).

Gutierrez-Domenech, M. (2005). Employment after motherhood: A European comparison. Labour Economics, 12, 1, S. 99-123. 
Han, W.-J., C. Ruhm \& Waldvogel, J. (2007). Parental leave policies and parents' employment and leave-taking. Bonn: Forschungsinstitut für die Zukunft der Arbeit (IZA Discussion Paper Nr. 324).

Hashimoto, M., R. Percy, T. Schoellner \& Weinberg, B. A. (2004). The long and short of it: Maternity leave coverage and women's labor market outcomes. Bonn: Forschungsinstitut für die Zukunft der Arbeit (IZA Discussion Paper Nr. 1207).

Henninger, A., Wimbauer, C. \& Dombrowski, R. (2008). Geschlechtergleichheit oder ,exklusive Emanzipation'? Ungleichheitssoziologische Implikationen der aktuellen familienpolitischen Reformen. Berliner Journal für Soziologie, 18, 1, S. 99-128.

Kluve, J. \& Tamm, M. (2013). Parental leave regulations, mothers' labor force attachment and fathers' childcare involvement: Evidence from a natural experiment, Journal of Population Economics, 26, 3, S. 983-1005.

Lalive, R. \& Zweimüller J. (2009). How does parental leave affect fertility and return to work? Evidence from two natural experiments, Quarterly Journal of Economics, 124, 3, S. 1363-1402.

Lalive, R., Schlosser, A., Steinhauer, A. \& Zweimüller, J. (2011). Parental leave and mothers' careers: The relative importance of job protection and cash benefits. Zurich: University of Zurich, Department of Economics (Working Paper No. 42).

Liu, Q. \& Nordstrom Skans, O. (2010). The duration of paid parental leave and children's scholastic performance, The B.E. Journal of Economic Analysis \& Policy, 10, 1, Article 3.

Merz, M. (2005). Women's hours of market work in Germany: The role of parental leave In: GomezSanchez, R. et al. (Hrsg.), Labor supply and incentives to work in Europe. Northhampton, MA: Edward Elgar Publishing,

Oláh, L. S. (2003). Gendering fertility: Second births in Sweden and Hungary. Population Research and Policy Review, 22, 2, S. 171-200.

Ondrich, J., Spiess, C. K. \& Yang, Q. (2003a). Changes in women's ages after parental leave. Schmollers Jahrbuch, 123, 1, S. 125-138.

Ondrich, J., Spiess, C. K., Wagner, G. G. \& Yang, Q. (2003b). The liberalization of maternity leave policy and the return to work after childbirth in Germany. Review of Economics of the Household, 1, 12, S. 77-110.

Ondrich, J., Spiess, C. K., Wagner, G. G. \& Yang, Q. (1999). Full Time or part time? German parental leave policy and the return to work after childbirth in Germany. Research in Labor Economics, 18, S. 41-74.

Ondrich, J., Spiess, C. K. \& Yang, Q. (1996). Barefoot and in a German kitchen: Federal parental leave and benefit policy and the return to work after childbirth in Germany. Journal of Population Economics, 9, 3, S. 247-266.

Pylkkänen, E. \& Smith, N. (2003). Career interruptions due to parental leave: A comparative study of Denmark and Sweden. Paris: Organization of Economic Co-operation and Development (Social, Employment and Migration Working Papers, DELSA/ELSA/WD/ SEM (2003) 1).

Riphahn, R. \& Bergemann, A. (2011). Female labour supply and parental leave benefits: The causal effect of paying higher transfers for a shorter period of time. Applied Economics Letters, 18, 1, S. 17-20.

Ristau, M. (2005). Der ökonomische Charme der Familie. Aus Politik und Zeitgeschichte, 23-24, S. 1622.

Ruhm, C. J. \&Teague, J. L. (1997). Parental leave policies in Europe and North America. In: Blau, F. \& Ehrenberg, R. (Hrsg.), Gender and family issues in the workplace. New York, NY: Russell Sage Foundation Press.

Ruhm, C. (1998). The economic consequences of parental leave mandates: Lessons from Europe, The Quarterly Journal of Economics, 113, 1, S. 285-317.

Ruhm, C. (2000), Parental leave and child health. Journal of Health Economics, 19, 6, S. 931-960.

Schober, P. (2011): Maternal labor market return, parental leave policies, and gender inequality in housework. Berlin: Deutsches Institut für Wirtschaftsforschung (SOEP Paper Nr. 422).

Schönberg, U. \& Ludsteck, H. (2007). Maternity leave legislation, Female labor supply, and the family wage gap. Bonn: Forschungsinstitut für die Zukunft der Arbeit (IZA Discussion Paper Nr. 2699). 
Spieß, C. K. (2011). Vereinbarkeit von Familie und Beruf - wie wirksam sind deutsche "Care Policies"? Perspektiven der Wirtschaftspolitik, 12, s1, S. 4-27.

Schulz, F. \& Rost, H. (2012). Hausarbeitsteilung und Erwerbsunterbrechung von Müttern unter den Bedingungen des neuen Elterngeldgesetzes. Erste empirische Befunde aus Bayern. Zeitschrift für Familienforschung/Journal of Family Research, 24, 1, S. 27-45.

Spiess, C. K. \& Wrohlich, K. (2008). The parental leave benefit reform in Germany: Costs and labour market outcomes of moving towards the Nordic model, Journal of Population Research and Policy Review, 27, 5, S. 575-591.

Steiner, V., K. Wrohlich, P. Haan \& Geyer, J. (2012). Documentation of the tax-benefit microsimulation model STSM: Version 2008. Berlin: Deutsches Institut für Wirtschaftsforschung (DIW Data Documentation Nr. 31).

Tanaka, S. (2005), Parental leave and child health across OECD countries, The Economic Journal, 115, 501, S. F7-F28.

Wagner, G. G., Frick, J. R., \& Schupp, J. (2007). The German Socio-Economic Panel Study (SOEP) Scope, evolution, and enhancements. Schmollers Jahrbuch, 127, 1, S. 139-169.

Wimbauer, C. \& Henninger, A. (2008). Magd des Marktes. Das Elterngeld und die neue Familienpolitik, Blätter für Deutsche und Internationale Politik, 53, 8, S. 69-76.

Wrohlich, K., Berger, E., Geyer, J., Haan, P., Sengül, D., Spieß, C. K. \& Thiemann, A. (2012). Elterngeld Monitor. Berlin: Deutsches Institut für Wirtschaftsforschung (DIW Politikberatung kompakt, Nr. 61).

Wrohlich, K. (2011). Labor supply and child care choices in a rationed child care market. Berlin: Deutsches Institut für Wirtschaftsforschung (DIW Discussion Papers 570).

Würtz Rasmussen, A. (2010). Increasing the length of parents' birth-related leave: The effect on children's long-term educational outcomes, Labour Economics 17, 1, S. 91-100.

Eingereicht am/Submitted on: 21.12.2012

Angenommen am/Accepted on: 25.07.2013

Anschriften der Autorinnen und Autoren/Addresses of the authors:

Dr. Johannes Geyer

Prof. Dr. Peter Haan

Prof. Dr. C. Katharina Spieß (Korrespondenzautorin/Corresponding author)

Dr. Katharina Wrohlich

Deutsches Institut für Wirtschaftsforschung (DIW) Berlin

(German Institut for Economic Research)

Mohrenstraße 58

10117 Berlin

Deutschland/Germany

E-Mail:jgeyer@diw.de phaan@diw.de kspiess@diw.de kwrohlich@diw.de 